bewegung entfernter Objecte bei Tage 25". Alle diese Daten scheinen zu beweisen, dass die Amplitude der Zitterbewegung der Fixsterne oder der Radius ihres Scintillationszerstreuungskreises im Allgemeinen einige Secunden beträgt.

Die Existenz des Scintillationszerstreuungskreises hat wohl in der praktischen Astronomie und insbesondere in Bezug auf die Beobachtung durch grosse Instrumente eine besondere Bedeutung. Wenn ein punktförmiger Fixstern als Kreis von mehreren Secunden Durchmesser erscheint, so muss dasselbe von den einzelnen Punkten eines aus. gedehnten Objectes, wie des Mondes oder eines Planeten gelten, und ein solches Object muss folglich bei Beobachtung durch ein Instrument von grosser Oeffnung undeutlich erscheinen, wenn die Atmosphäre sich in einem, dem Entstehen der Scintillation günstigen Zustande befindet. Letzteres ist beispielsweise bei Wien stets der Fall; bei zahlreichen, $z \mathrm{u}$ verschiedenen Zeiten angestellten Beobachtungen habe ich im Scintilloskope niemals Abhandensein der Scintillation wahrgenommen. Diese Verundeutlichung der Bilder in grossen Instrumenten durch den Einfluss der Scintillation ist iberdies $u m$ so beträchtlicher, je stärker die Vergrösserung des Instrumentes ist, denn der Durchmesser des Scintillationszerstreuungskreises unterliegt der Vergrösserung des Instrumentes. Jedem Orte kommt eine bestimmte Scintillationsconstante $\mathrm{zu}$, nämlich der mittlere Grad der Scintillation daselbst, welcher durch ein Scintillometer bestimmt werden kann und für verschiedene Orte sehr verschieden ist. $\mathrm{Da}$ andererseits diese Scintillationsconstante vom grössten Einflusse auf die Brauchbarkeit eines grossen Instrumentes ist, so scheint es, dass bei der Auswahl eines Ortes zur Aufstellung eines solchen Instrumentes vorhergegangene Scintillometerbeobachtungen von Nutzen sein könnten. Die Nützlichkeit solcher Beobachtungen erscheint mir um so einleuchtender, als zwar die Abhängigkeit des Grades der Scintillation von der Höhe des Beobachtungsortes bisher durch keine Messungen festgestellt ist, ich aber durch noch nicht publicirte Beobachtungen zur Kenntniss gelangt bin, dass die Scintillation der Sterne vorwiegend in den unteren Luftschichten entsteht.

Ein sehr transportables und empfindliches Scintilloskop ist das Marius'sche Dasselbe besteht einfach aus einem Fernrohre von 3 oder mehr Zoll Oeffnung, dessen Ocular eingeschoben worden ist, so dass jeder leuchtende Punkt als eine grosse helle Scheibe erscheint. Die Scintillation verursacht sehr charakteristische Fluctuationen der Helligkeit und Farbe innerhalb dieser hellen Scheibe, Abhandenseir der Fluctuationen bedeutet Abhandensein der Scintillation. Ein solches Scintilloskop ist hinlänglich empfindlich, so dass. ich mit einem solchen bei einer torrestrischen Lichtquelle schon in einer Entfernung von 20 Schritten Scintillation constatiren konnte.

Die Ungleichmässigkeiten der Atmosphäre verursachen also eine Verundeutlichung der Bilder ausgedehnter Objecte in den Fernrohren, indem jeder Punkt als ein Kreis, Scintillationszerstreunngskreis, erscheint, und zwar ist die Verundeutlichung um so beträchtlicher, je stärker die Vergrösserung des Instrumentes ist, trifft also am empfindlichsten die grossen Instrumente. Der mittlere Radius des Scintillationszerstreuungskreises eines Ortes lässt sich mittelst eines Scintillometers bestimmen, wie ich an anderem Orte gezeigt habe, und mittelst eines Scintilloskopes schätzen. DasScintillometer gestatiet also die Eignung eines Ortes zur Aufstellung eines grossen Instrumentes vorherzubestimmen Wien I 887 Mai.

\title{
Beobachtungen des Cometen 1886 III
}

angestellt mit dem Zehnzöller der Sternwarte $z$ u Genf.

\begin{tabular}{|c|c|c|c|c|c|c|c|c|c|c|}
\hline I $886^{\circ}$ & M.Z.Genf & $\Delta \alpha$ & $\Delta \delta$ & Vgl. & c app. & $\log p \cdot d$ & $\delta$ app. & $\log p \cdot A$ & Red. ad 1.app. & * \\
\hline Mai & $\begin{array}{l}\mathrm{I} 5^{\mathrm{h}} \mathrm{I}^{\mathrm{I}^{\mathrm{m}}} \mathrm{I}^{\mathrm{S}} \\
1440 \text { 2 }\end{array}$ & $\begin{array}{l}-0^{m_{2}} 3^{\mathrm{s}} .02 \\
+143.13\end{array}$ & $\begin{array}{r}8^{\prime} 6.8 \\
-\quad 350.7\end{array}$ & $\begin{array}{l}\text { I } 3.8 \\
\text { I } 8.6\end{array}$ & $23^{\mathrm{h}} 13^{\mathrm{m}} 5^{15} \cdot 7 \mathrm{I}$ & $\begin{array}{l}9.658 \mathrm{n} \\
9.675 \mathrm{n}\end{array}$ & $\begin{array}{c}+28^{\circ} 22^{\prime} 49^{\prime \prime} 2 \\
-\end{array}$ & $\begin{array}{l}0.703 \\
0.725\end{array}$ & $\begin{array}{l}+0.15-7.0 \\
+0.17-7.5\end{array}$ & \\
\hline
\end{tabular}

Mittlere Oerter der Vergleichsterne für $\mathbf{1 8 8 6 . 0 .}$

\begin{tabular}{|c|c|c|c|}
\hline$*$ & $a \pm 886.0$ & $\delta$ I $886 \circ$ & Autorität \\
\hline $\begin{array}{l}\text { I } \\
2\end{array}$ & $\begin{array}{l}23^{\mathrm{h}} 14^{\mathrm{m}} \times 4^{\mathrm{s}} \cdot 5^{8} \\
231522\end{array}$ & $\begin{array}{l}+28^{\circ} 14^{\prime} 49^{\prime \prime} .4 \\
+30\end{array}$ & $\begin{array}{l}W_{2} 23^{\mathrm{h}} \cdot 257 \\
\mathrm{DM}+20^{\circ}\end{array}$ \\
\hline
\end{tabular}

Be mierkungen.

Mai 3. Hübsche Abbildung eines grossen Cometen. Sehr heller Schweif, dünn am Kopfe und sich dann in Form eines türkischen Säbels allmälig ausbreitend; Krümmıng nach Süden. Schweiflänge I5'. Heftiger Nordwind.

* 4. Comet ist nicht mehr so schön wie gestern; der beim Kern gelegene Schweiftheil ist breiter geworden und nicht mehr so scharf. Positionswinkel des Schweifes am Kopfe ganz nahe $270^{\circ}$.

\section{A. Kammermann.}

